Dossiê - Para além do “ativismo judicial" e da “judicialização da política" DOI: $10.5433 / 2176-6665.2016 \mathrm{v} 21 \mathrm{~N} 1 \mathrm{P} 22$

\title{
La oralidad. Nueva Perspectiva de la Justicia en MÉxICo
}

\author{
ANGÉLICA CuÉllar VÁZqueZ ${ }^{l}$
}

\section{RESUMEN}

La Reforma Constitucional en Materia Penal de 2008 modificó la aplicación de justicia en México. La transición entre el sistema inquisitivo y el sistema acusatorio oral resignificó las prácticas de los operadores jurídicos. La oralidad es el eje central del nuevo sistema de justicia. Con la oralidad como eje se busca que el nuevo sistema sea más garantista y respete los derechos humanos de la víctima y del imputado.

Palabras clave: Oralidad. Transición. Resignificación de prácticas. Operadores jurídicos. Sistema garantista.

\section{A oralidade. Nova perspectiva sobre a Justiça no México}

\section{Resumo}

A Reforma Constitucional em Matéria Penal de 2008 modificou a aplicação da justiça no México. A transição entre o sistema inquisitivo e o sistema acusatório oral ressignificou as práticas dos operadores jurídicos. A oralidade é o eixo central do novo sistema de justiça. Através da oralidade como eixo pretende-se que o novo sistema seja mais garantista e respeite os direitos humanos da vítima e do imputado. Palavras-chave: Oralidade. Transição. Ressignificação de práticas. Operadores jurídicos. Sistema garantista.

1 Doctora en sociología y profesora titular de la Facultad de Ciencias Políticas y Sociales de la Universidad Nacional Autónoma de México (UNAM), México. acuellarunam@gmail.com 


\section{INTRODUCCIÓN ${ }^{2}$}

Para entender la transición que el país vivió en el año de 2008 con respecto al nuevo sistema de justicia, es necesario explicar las circunstancias que se dieron para cambiar del sistema inquisitivo al sistema acusatorio oral.

La transición comenzó a gestarse desde que el Partido Revolucionario Institucional (en adelante PRI) dejara la presidencia del país después de setenta años en el poder, para dar paso al Partido Acción Nacional (en adelante PAN) en el año 2000. A partir del 2000 y hasta el 2012 el PAN se mantuvo en la presidencia de México, con los gobiernos de Vicente Vox Quesada (2000-2006) y Felipe Calderón Hinojosa (2006- 2012) respectivamente. Es justo en medio de esta transición política, que se observó la posibilidad de modificar el sistema de justicia del país. Esto se puede explicar porque el nuevo gobierno pretendió consolidar el cambio presidencial a partir del aspecto de seguridad nacional. Este fue la principal prioridad para estos dos mandatos, especialmente el de Felipe Calderón Hinojosa a partir del año 2006.

El factor político fue un elemento que posibilitó la creación de una nueva reforma para el sistema de justicia, pero no fue el único. El otro factor que influyó para que los gobiernos comenzaran a poner sobre la mesa la discusión de la reforma fue el incremento de la inseguridad y la delincuencia, en específico el poder que el crimen organizado comenzó a tener a principios de la década de los noventa y que con el paso del tiempo aumentó. La percepción de la inseguridad y

2 Para la realización de este artículo agradezco la colaboración de Analy Loera, ayudante de investigación del Consejo Nacional de Ciencia y Tecnología (CONACyT). Este trabajo forma parte de las investigaciones apoyadas por la Dirección General de Asuntos del Personal Académico (DGAPA) de la UNAM, es parte del Programa de Apoyo a Proyectos de Investigación e Innovación Tecnológica (PAPITT) IN 305915, El nuevo arreglo institucional desde las prácticas de los operados jurídicos. La reforma de justicia penal en el estado de Morelos. 
la delincuencia que la sociedad tenía en aquella década provocó que se perdiera la confianza en las instituciones de procuración e impartición de justicia.

Guillermo Zepeda Lecuona (2011) en su texto Crimen sin castigo. Procuración de justicia penal y ministerio público y el libro de Arturo Alvarado Mendoza El tamaño del infierno. Un estudio sobre la criminalidad en la Zona Metropolitana de la Ciudad de México, dan cuenta de la gravedad de la situación. Alvardo Mendoza (2012, p. 49) describe el fenómeno de la criminalidad en México de la siguiente manera:

[...] la criminalidad en México es producto de la evolución histórico-social de los delitos, del trabajo de las policías, de los agentes gubernamentales (del Estado mexicano) y de las respuestas sociales a los delincuentes y las políticas del Estado en el siglo XX. Pero también obedece a su particular historia política.

Estos autores plantean la problemática de la inseguridad y la delincuencia en México, proporcionan datos estadísticos donde se puede observar este fenómeno y la relación que tiene con los operadores jurídicos y las instituciones de justicia.

En los últimos veinte años, el panorama delictivo en México se ha transformado de manera profunda. El número de ilícitos reportados a las autoridades, había presentado un incremento sustancial durante los años ochenta (principalmente debido a las actividades vinculadas con el narcotráfico) experimentó un aumento sin precedentes a mediados de los noventa. En apenas una década, México pasó de ser un país con criminalidad media, a presentar una incidencia delictiva particularmente alta, y cuyos indicadores de violencia lo ubican entre las diez naciones más violentas del mundo. Este notable incremento está estrechamente asociado con variables sociales (como urbanización y el aumento de la drogadicción) y económicas (como el desempleo, la distribución del ingreso y el descenso en las expectativas a raíz de la crisis financiera del 1994-1995). Además, México ha visto la consolidación del crimen organizado, que ha desbordado 
el ámbito delictivo tradicional del narcotráfico y ahora conforma numerosas bandas dedicadas al secuestro, la sustracción de infantes o el robo de vehículos, así como modalidades muy complejas, como delitos informáticos y el tráfico de órganos (ZEPEDA LECUONA, 2011, p. 14).

El factor político y el factor de la inseguridad y delincuencia visibilizaron los vicios y deficiencias que el sistema inquisitivo de impartición de justicia tenía desde hacía mucho tiempo. La reestructuración del sistema tuvo por objetivo erradicar las deficiencias y vicios de los operadores jurídico. Así lo plantea Alvarado Mendoza (2012, p. 63-64):

La noción de delincuencia no puede ser la de un concepto abstracto jurídico, ahistorico y asocial. La criminalidad es un constructo político y social colectivo, históricamente construido, producto de la lucha entre individuos y grupos por imponer un orden estatal-jurídico, una república donde imperen normas y nociones de justicia (algunas de ellas siempre estarán en competencia), mediante procesos tanto negociados como impositivos. El crimen está vinculado con los esfuerzos del Estado por imponer un orden a través de mecanismos legales y regulatorios.

En el año 2004 en el gobierno de Vicente Fox Quesada se presentó la primera iniciativa para la Reforma Constitucional en Materia Penal (en adelante RCMP). Este fue el primer paso de muchos que se tendrían que dar para que la RCMP se aprobara en el año de 2008. Es importante señalar que la iniciativa propuesta por Vicente Fox no progresó, solo se decretó la Ley de Seguridad Publica el 31 de enero de 2005.

En el año de 2006 Felipe Calderón también del PAN, asumió la presidencia de la República. El gobierno de Felipe Calderón se enfocó en la seguridad nacional, esa fue su prioridad durante los seis años de su mandato. La idea de seguridad nacional que Felipe Calderón tenía se plasmó en el Plan Nacional de Desarrollo 2006-2012 (en adelante PND) (MÉXICO, 2012). Se llevaron iniciativas de ley frente a la Cámara 
de Diputados y la Cámara de Senadores. De todas las iniciativas presentadas ante el pleno del Poder Legislativo, la del 25 de abril del 2007 fue la más contundente, pues a raíz de esta iniciativa se produjo el cambio de sistema de justicia en el país. El 28 de mayo de 2008 se aprobó en ambas cámaras y se envió al ejecutivo para su posterior publicación. Finalmente el 18 de junio de 2008 la reforma se publicó el Diario Oficial de la Federación (en adelante DOF). La homologación de la RCMP en el país se realizará con fecha límite del 18 de junio de 2016, es decir, para esta fecha todos los estados de la República tendrán que haber finalizado la transición al sistema acusatorio oral.

Si bien el camino para llegar a una RCMP ha sido largo y complicado por todas las discusiones que se dieron en el Poder Legislativo, la aplicación del nuevo sistema de justicia es la que presentó más dificultad; es un sistema que se basa en la oralidad para desarrollar todos y cada uno de los pasos del proceso. La oralidad es el punto distintivo entre el nuevo y el viejo sistema.

Este artículo tiene por objetivo observar como la oralidad se vuelve un factor importante en el nuevo sistema de justicia en México. Por lo tanto se recurre a la comparación del sistema inquisitivo con el sistema acusatorio oral. También se expone como los operadores jurídicos adaptaron sus antiguas prácticas e incorporaron las nuevas prácticas que la RCMP les impuso. Por esta razón se habla de una reconfiguración de prácticas por parte de los operadores, pero también se explican las modificaciones estructurales que se realizaron desde la ley, y cuáles son los roles que les corresponden a cada operador: Ministerio Público (en adelante MP), Abogado Defensor Público (en adelante ADP) y Juez.

Todo lo anterior abrirá las puertas para comprender la nueva visión de justicia en México. Una visión que en México nunca se tuvo, un sistema que se propone ser más garantista y apegado a los derechos humanos. 


\section{El Cambio: Del Sistema Escrito al Sistema Oral}

El cambio de un sistema inquisitivo a un sistema acusatorio oral, es un proceso complejo; supone no solo la modificación de las prácticas de los operadores jurídicos, también el cambio de la estructura del Poder Judicial. Este apartado presenta el cambio de roles de los operadores jurídicos que la RCMP originó en 2008.

El sistema inquisitivo fue el sistema de justicia por excelencia en México durante mucho tiempo. El sistema inquisitivo se basa en la presunción de culpabilidad del detenido, siendo el inculpado quien tenía que probar su inocencia. Bajo esta lógica es cómo funcionaba el sistema de justicia en el país. La estructura principal de este sistema se basaba en tres figuras: la Defensoría, Ministerio Público y el Juez. La Defensoría estaba representada por el ADP, quien asesoraba al inculpado dentro del proceso penal. El Ministerio Público era quien asesoraba a la víctima en el proceso penal, además integraba el expediente de investigación que se enviaba al Juez para su revisión y así obtener un dictamen sobre el caso. La última pieza del sistema inquisitivo es el Juez, que en la mayoría de los casos se encontraba ausente en todo el proceso. El Juez contaba con un secretario proyectista, el cual tenía la función de leer el expediente que el MP le proporcionaba y con ello realizaba un proyecto de sentencia, ya se absolutoria o condenatoria, se la presentaba al Juez, éste revisaba y avalaba o rechazaba el proyecto de sentencia; nunca tenía contacto ni la víctima y ni el inculpado.

Esta era la lógica que pautaba el sistema inquisitivo, un sistema que le otorgaba bastante injerencia al MP, ya que era quien realizaba la investigación, y también quien decidía cual era el contenido del expediente. El proceso de la averiguación previa solo recaía en el MP, con la ayuda de los cuerpos policiacos, de los peritos y las declaraciones de los testigos; existía gran hermetismo con respecto al 
proceso y la investigación. Finalmente el ADP no tenía ningún peso en el proceso, su función se limitaba a informar al inculpado del proceso y el dictamen final que el Juez hizo sobre el caso.

La modificación en el sistema de justicia, originó que los roles de los operadores sufrieran un reacomodo. La estructura del sistema acusatorio oral es muy diferente. Para la impartición y procuración de justicia en el sistema acusatorio oral, se definieron los siguientes roles para los operadores:

- El Juez de control: encargado de recopilar las pruebas y ver si el caso procede a juicio o no.

$-E l$ Juez de juicio oral: tiene la función de guiar y presenciar la confrontación de las partes involucradas (víctima e imputado representados por el MP y el ADP).

- El Juez de ejecución: es el encargado de dictaminar la sentencia de un caso a partir de lo expuesto en las audiencias de juicio oral.

- MP: Se encarga de integrar de la carpeta de investigación y presentarla en las audiencias de juicio oral. También es el encargado de velar por los derechos de la víctima, recabando pruebas para demostrar la culpabilidad del imputado.

$-A D P$ : su principal función es defender al imputado. Puede conocer el contenido de la carpeta de investigación, además tiene que estar presente en las audiencias de juicio oral.

- Policía ministerial: El cuerpo policiaco es el encargado de realizar la detención del imputado y recopilar testimonios en el lugar del hecho.

- Peritos: Este personal trabaja para la Fiscalía y aporta pruebas científicas al caso, recopilan pruebas del lugar de los hechos y realizan pruebas médicas que son canalizadas al MP para que las incorpore a la carpeta de investigación.

- Especialistas en Métodos Alternativos: Cuando se estable la transición de sistema de justicia penal, se incorpora una nueva 
dependencia para la resolución de conflictos: el Centro de Justicia Alternativa, donde se canalizan casos que son denominados no graves por la ley. Dentro de este Centro la dinámica no es igual que la que se lleva en el proceso penal. La resolución de conflictos por medio de mecanismos alternativos requiere de Facilitadores, Mediadores y Conciliadores, quienes son los operadores jurídicos que intervienen entre la persona que comete un delito y la que recibe el daño, de tal manera que se pueda llegar a un acuerdo reparatorio donde ambas partes queden conformes, sin la necesidad de entablar acción legal en contra de la persona que cometió el daño.

Como se puede observar, la estructura que tiene el sistema inquisitivo y el sistema acusatorio es distinta; los agentes involucrados en uno y en otro tienen funciones diferentes. En el nuevo sistema se hace hincapié que los operadores deben de respetar el debido proceso, y estar en todo momento involucrados en el desarrollo del mismo. Las audiencias de juicio oral son públicas, es decir se realizan con presencia de público. Ahora cualquier persona puede estar presente en una audiencia. Este elemento no se encontraba en el antiguo sistema, antes todo era hermético; la interacción entre los operadores jurídicos era casi nula.

Si bien la transición se enfocó a crear un sistema más garantista, también se consiguió hacer más visibles a todos los involucrados en el proceso, dejando que la oralidad sea muy importante para la construcción de la verdad jurídica.

\section{Capacitación de Los Operadores Jurídicos}

Como ya se ha mencionado la RCMP posibilitó la creación de nuevas figuras jurídicas y nuevas funciones para los operadores, también buscó fortalecer las que ya se tenían en el antiguo sistema mediante la capacitación. Por lo tanto hablar sobre la capacitación que los agentes recibieron es importante. El objetivo de la capacitación no 
fue solo para llevar el debido proceso en las audiencias, sino establecer el contacto que los operadores jurídicos tienen con la víctima, el imputado y los testigos en general. Lo que se conoce en la ley como principio de contradicción.

La capacitación se basó en un diagnostico que las instituciones hicieron sobre las deficiencias y vicios que los operadores tenían. A partir de este diagnóstico se elaboraron cursos de capacitación con el objetivo de dotar de nuevas herramientas a los operadores. Cursos sobre el lenguaje oral y corporal, técnicas de interrogación y contrainterrogación. La capacitación pretende reconfigurar las prácticas de los operadores. En este sentido se busca un nuevo perfil de operadores.

Tener operadores mejor capacitados supone que el proceso penal sea más eficiente y transparente, de tal modo que se pueda recuperar la confianza en las instituciones de justicia. El sistema inquisitivo había desgastado la relación entre el Poder Judicial y la sociedad; la población no confiaba en sistema justicia.

El trabajo empírico se realizó en el estado de Morelos, específicamente en la ciudad de Cuernavaca, capital del estado. La principal razón de por qué se centró el análisis en este estado, es porque fue uno de los pioneros en la implementación del nuevo sistema oral. Se instrumentó desde 2008. En este sentido se puede observar cómo se lleva a cabo la aplicación del nuevo sistema.

Las condiciones legales para poner en marchar la RCMP, están puntualizadas en el Código Nacional de Procedimientos Penales (en adelante CNPP), publicado el 5 de marzo de 2014 en el DOF. El CNPP permite saber cómo debe de ser el procedimiento de los operadores dentro del sistema (MÉXICO, 2014).

A partir de la publicación de RCMP, se creó un organismo administrativo cuya función principal es apoyar a las autoridades federales y locales en todo lo relacionado con la implementación 
del nuevo sistema de justicia, es decir, los cambios estructurales sobre la infraestructura, la definición de roles de los operadores, los posibles cambios normativos que el proceso requiera, la capacitación de los operadores y la difusión del sistema acusatorio oral. Este organismo es la Secretaría Técnica del Consejo de Coordinación para la Implementación del Sistema de justicia Penal (en adelante SETEC) (MÉXICO, 2014). La SETEC presentó alrededor de quince programas con el objetivo de capacitar a distintos operadores.

Los operadores que formaron parte de estos programas de capacitación son los siguientes:

- Jueces y Magistrados

- Administrador de la Sala

- Agente del Ministerio Público

- Agentes del Ministerio Público Ordenador

- Defensores

- Especialistas en Métodos Alternos

- Policía de Investigación

- Policía

- Peritos

- Personal del sistema Penitenciario

- Asesor Jurídico de Víctimas

- Periodistas y Personal de Comunicación Social

- Abogados Litigantes

- Personal de las Unidades Especializadas contra el Secuestro

- Capacitación Docente (MÉXICO, 2014).

La SETEC presentó un programa importante el 29 de abril de 2010 denominado Programa de Capacitación y Difusión de la SETEC. El programa tuvo como finalidad definir los objetivos y estrategias del PND de 2006-2012. Este programa contiene los ejes principales para la capacitación de los operadores. 
El PND tiene como objetivos primordiales, profesionalizar a los tres principales operadores (MP, ADP y Juez), mejoramiento de las instalaciones penitenciarias, garantizar una justicia pronta y expedita para la víctima y para el imputado, promover los procesos orales, promover los mecanismos alternativos que plantea este nuevo sistema y como ya se mencionó combatir los vicios en las prácticas de los operadores jurídicos. El programa de capacitación utilizó las ideas expuestas en el PND, de tal forma que se plantearon los siguientes tres objetivos del programa:

- Sensibilización. Este objetivo tiene la misión de concientizar a los involucrados y a la población en general sobre la transición del sistema de justicia.

- Interiorización. Este segundo objetivo se enfoca a que los operadores reciban la capacitación adecuada dependiendo de la función que desempeñan dentro del nuevo sistema de justicia. Se divide principalmente en tres aspectos: el primero es una convocatoria para los nuevos operadores de justicia y la correspondiente capacitación. El segundo es la realización de un curso introductorio por los aspirantes que previamente han sido seleccionados (Jueces, MP, ADP, cuerpos policiales, peritos y mediadores). El tercero es la futura capacitación de operadores con base en los requerimientos que la RCMP necesite para la implementación del sistema acusatorio oral.

- Aplicación. Este último objetivo tiene como finalidad poner en práctica todos los elementos que se presentaron en los cursos de capacitación. Fue así como los operadores obtuvieron las habilidades, destrezas y conocimientos necesarios para aplicar el nuevo sistema de justicia. También se continúa con la creación de foros, mesas redondas y claustros académicos de retroalimentación 
con el fin de que se mantenga una constante actualización por parte de los operadores.

Las instituciones que aplicaron esta capacitación fueron las siguientes: las Secretarías de Seguridad Pública, las Procuradurías Generales de Justicia, la Defensorías Públicas y las áreas en las que existen operadores jurídicos que intervienen directa o indirectamente.

Como se puede observar, la capacitación de los operadores tiene un peso importante dentro del nuevo sistema justicia. También se buscó que los operadores promuevan los medios alternos de resolución de conflictos y también los juicios orales. La capacitación hace que los operadores usen la oralidad como una nueva herramienta.

Para poder entender mejor la situación que tienen los operadores en el nuevo campo jurídico, es necesario utilizar una corriente teórica que permita observar como los operadores resignifican sus prácticas a partir de las modificaciones institucionales. La teoría relacional de Pierre Bourdieu logra establecer las pautas para observar la relación entre el campo jurídico y las prácticas de los operadores. Los conceptos de Bourdieu usados son campo y habitus. Bourdieu e Wacquant (2005, p. 150) plantea que el campo es:

Una red o una configuración de las relaciones objetivas entre posiciones. Estas posiciones están objetivamente definidas, en su existencia y en las determinaciones que imponen sobre sus ocupantes, agentes o instituciones, por su situación presente y potencial en la estructura de distribución de especies de poder, cuya posesión ordena el acceso a ventajas específicas que están en juego en el campo, así como por su relación objetiva con otras posiciones.

Es imposible mencionar el concepto de campo y no hablar del habitus, ya que son conceptos que van de la mano. El concepto de habitus es la encarnación o materialización del campo, así lo explica Bourdieu (2007, p. 86): 
El habitus es un Sistema de disposición duraderas y transferibles, estructuras estructuradas predispuestas a funcionar como estructuras estructurantes, es decir, como principios generadores y organizadores de prácticas y de representaciones que pueden ser objetivamente adaptadas a su meta sin suponer el propósito consciente de ciertos fines ni el dominio expreso de las operaciones necesarias para alcanzarlos, objetivamente 'reguladas' y 'reguladores' sin ser para nada el producto de la obediencia a determinadas reglas, y por todo ello, colectivamente orquestadas sin ser el producto de la acción, organizada de un director de orquestas.

Siguiendo lo expuesto por Bourdieu, sus conceptos facilitan el análisis sobre la resignificación de prácticas por parte de los operadores en el nuevo sistema de justicia.

\section{LA ORALIDAD COMO CONSTRUCCIÓN DE LA VERDAD JURÍDICA. LA PERCEPCIÓN DE LOS OPERADORES}

La percepción de los operadores jurídicos sobre el nuevo sistema de justicia permite entender cómo se posicionan dentro del campo jurídico y como están resignificando sus prácticas. Para poder observar dicha percepción, fue necesario hacer varias entrevistas a algunos operadores jurídicos (jueces, ministerios públicos y abogados defensores públicos). Las entrevistas se llevaron a cabo en la ciudad de Cuernavaca. A raíz del análisis de las entrevistas realizadas, se observó que la lucha por la verdad jurídica es lo que los operadores percibieron como distinto entre el nuevo y viejo sistema de justicia a partir de la construcción de la verdad jurídica o también llamada por ellos como certeza jurídica en cada sistema.

El debate principal que se presenta en la transición del sistema de justicia de acuerdo con las entrevistas es la construcción de la verdad jurídica. La construcción de la verdad jurídica parte de supuestos muy distintos entre un sistema y otro. En este apartado se observó como el 
sistema inquisitivo construyó la verdad jurídica a partir de lo escrito en el expediente comparándolo con el nuevo sistema acusatorio oral que construye la verdad jurídica a partir de la oralidad y el principio de contradicción.

En el sistema inquisitivo la certeza jurídica se establecía en el expediente escrito que el MP armaba. El expediente contenía todo lo recabado por los cuerpos policiales, peritos, declaraciones de la víctima, inculpado y los testigos. Lo que se encontraba en el expediente servía para construir la verdad. El expediente de investigación era enviado por el MP al Juez para que este pudiera elaborar la sentencia condenatoria o absolutoria solo basando en el expediente; aquí no se tenía confrontación cara a cara. La sentencia la hacía el secretario proyectista.

[...] antes bien fácil el juez no sabía ni que firmaba, y firmaba lo que decía su secretario proyectista, o el que le hacía la sentencia, porque nunca había percibido el juicio. $\mathrm{Ni}$ siquiera conoció al imputado. (Entrevistas hechas en Cuernavaca, Morelos, México, Juez 4, 2014).

Siguiendo esta lógica, se entiende que el MP tenía un peso importante en el antiguo sistema, ya que al construir el expediente, también construía la verdad jurídica. La discrecionalidad del MP en los procesos jurídicos penales, hizo que el desarrollo del proceso del antiguo sistema se volviera hermético con respecto a la aportación de pruebas, que en la mayoría de los casos eran pruebas tasadas (son aquellas pruebas que ya tienen un valor predeterminado por un operador jurídico). Al no haber nadie que vigile el trabajo que realizaban, se volvió un blanco fácil para la corrupción y la impunidad a la hora de armar el expediente de investigación. Estos eran algunos de los vicios del antiguo sistema.

Entonces, yo creo que a donde debe tener el gobierno cuidado para fortalecerlo y que esto resulte es en la institución del mp. Principalmente porque es la que le va 
a dar los hechos. La que le va a dar los elementos al juez para que éste determine una cosa $u$ otra. Yo creo que la debilidad más grande y la fortaleza que puede tener este sistema es en la procuración de justicia. En la procuraduría, porque son los que investigan. Son quienes prueban, quien ofrece las pruebas al juez y son quien ellos...manejan la información (Entrevistas hechas en Cuernavaca, Morelos, México, Juez 4, 2014).

El hecho que las pruebas fueran tasadas y no hubiera ratificación de las mismas por parte de los involucrados en el proceso, hacía que el sistema recayera en gran medida en los manos de los MP. En este sentido los operadores principales en el sistema inquisitivo fueron el MP y el secretario proyectista, ya que eran los encargados de construir la verdad jurídica a partir del expediente.

Las prácticas del MP en el antiguo sistema y en el nuevo casi no se han visto trastocadas por la reforma. Sin embargo, el MP tiene que mostrar todo el contenido de la carpeta de investigación (antes llamado expediente de investigación) ante la audiencia de juicio oral. Esto hace que la discrecionalidad del MP disminuya a comparación del sistema inquisitivo.

Por otro lado, el trabajo del ADP era casi nulo en el sistema de justicia inquisitivo. La invisibilización de este operador, deja ver la falta de igualdad entre los operadores que existía en el viejo sistema. El trabajo del ADP era esperar la sentencia que el Juez firmaba, elaborada por el secretario proyectista a partir del expediente que le entregaba el MP. Cuando la sentencia era condenatoria, y vale la pena señalar que en su mayoría las sentencias eran condenatorias, al inculpado le quedaba el camino de la apelación.

Por estas razones el operador jurídico más beneficiado por el cambio de sistema de justicia es el ADP, ya que ahora se ha vuelto visible no solo para los demás operadores, sino para las instituciones y la sociedad. La participación del ADP en el proceso de juicio oral se 
volvió fundamental, pues el nuevo sistema pretende ser más garantista tanto con la víctima como con el imputado. Se trata de equiparar a la víctima con el imputado, al MP con el ADP esa igualdad ayuda a que el abogado pueda realizar su trabajo de la mejor manera posible. Los abogados defensores de oficio han recibido también muchos cursos de capacitación. También es importante señalar que el ADP tiene contacto con la carpeta de investigación, es decir puede revisar todas las pruebas que el MP presente en la carpeta.

Yo que ya estuve en el sistema anterior, le puedo decir, que
antes íbamos prácticamente con una prueba tasada, que ya
lo que estaba por escrito era lo que daba la certeza al juez
para dictar una sentencia. Venía del ministerio público. Si,
del policía y unas acotaciones que hacía la propia fiscal. Si
bien es cierto que ahora existe lo mismo en la carpeta de
investigación, pero ya para poder llevarla a juicio tendría
que venir esa prueba de manera personal. Eso es lo que
vemos favorable en este nuevo sistema, porque entonces
ya tenemos más certeza, de que si existió ese testigo, de
que si existió esa evidencia, de que si existió esa detención
(Entrevistas hechas en Cuernavaca, Morelos, México, ADP
1 y 2,2014 ).

Con base en lo expresado por algunos operadores, se pude observar que se tiene mayor certeza en el desarrollo del proceso de juicio oral; se pude decir entonces que el nuevo sistema de justicia acusatorio oral es más transparente y con menos posibilidades de caer en prácticas corruptas; ahora todo tiene que ser de manera presencial ante el juez. En el antiguo sistema no se tenía la certeza de los testigos, ni de las pruebas; bastaba con que estuviera escrito en el expediente para que el juez lo diera por hecho que era verdad. "Ahora se juzgan a las personas y no al papel" es lo que dice un juez que fue entrevistado en el estado de Morelos. Por lo tanto el proceso se vuelve más humano, donde la interacción cara a cara entre los involucrados es importante para la valoración que hace el juez. 
Otra característica que los operadores prescriben en comparación con el antiguo sistema, es la rapidez con la que se resuelven los casos. Los trámites se volvieron más eficaces. Lo que en el antiguo sistema podría durar seis meses, un año o más para concluir un proceso, en el sistema acusatorio tarda menos, es decir, los tiempos procesales se redujeron.

Pues yo creo que es más rápido ¿no? de forma oral, antes todo era escrito, consignaban la averiguación ante el juez, el juez tenía que analizar, volver a revisar, ahora es de manera oral. Así es, todo es más fácil, muchísimo más rápido, en esa audiencia exponer todas tus pruebas y ya le das una idea al juez de que es lo que en las diligencias se tiene que realizar y es más rápido que ellos puedan tomar una decisión en ese caso [...] Todos los procedimientos son muchísimo más rápidos, tenemos salidas alternas y acuerdos reparatorios, es más fácil, tienen solución más pronta. (Entrevistas hechas en Cuernavaca, Morelos, México, MP 2, 2014).

Si bien los operadores expresan que la transición a un nuevo sistema en su mayoría favorece en general a todos los involucrados en el desarrollo del proceso, también hacen notar que existe resistencia al cambio. Las principales resistencias se encuentran en las personas que tienen una trayectoria amplia en el sistema inquisitivo.

[...] la transición es difícil, lo va a ser porque es un cambio de paradigma. México estaba acostumbrado a un sistema totalmente escrito...pero la transición es muy paradigmática, muchas opiniones encontradas, más de los abogados que se han dedicado todo el tiempo al área penal, porque les quitan el expediente. Porque los jueces no tienen conocimiento del expediente. Los mismos de los juzgados federales no lo aceptan. (Entrevistas hechas en Cuernavaca, Morelos, México, Juez 4, 2014)

En el caso del Juez, el cambio de sistema modificó sus antiguas prácticas de manera importante, antes era un juez ausente, ahora tiene que estar presente en las audiencias. En el sistema inquisitivo 
el Juez tenía pleno acceso al expediente, en el sistema acusatorio no es así; el Juez desconoce por completo el contenido de la carpeta de investigación. Esto es algo con lo que los jueces tienen que lidiar pues solo conocerán el contenido de la carpeta en la audiencia y lo expuesto por el MP y el ADP. El MP aun tiene un peso importante, se puede decir que es el orquestador tanto en el antiguo sistema como del nuevo sistema de justicia.

Yo no sé qué tiene la carpeta de investigación. O todas las pruebas que tenga la carpeta de investigación si el mp no me las dice. Y si él las esconde y al defensor, pues le conviene, pues yo ya no supe nada. (Entrevistas hechas en Cuernavaca, Morelos, México, Juez 4, 2014)

A pesar de ello, el sistema acusatorio posibilita que todas las partes tengan una participación dentro del proceso con menor desigualdad. Todo lo anterior lleva a pensar que el punto de intersección entre los tres operadores que se entrevistaron, es que el sistema acusatorio oral es más garantista que el sistema inquisitivo y que supone mayor equidad entre dos de ellos: el MP y el ADP.

\section{La Materialización de la Reforma y la Oralidad. Las Audiencias de Juicio Oral}

El escenario donde se desenvuelven los operadores en el sistema de justicia oral son las salas de audiencia. Es ahí donde se interrelacionan los operadores, la víctima, imputado y los testigos.

Para observar este escenario fue necesario buscar un autor que permitiera abordar esta interacción. En este sentido, Goffman y su teoría sobre la ritualidad permite analizar la interacción en las audiencias.

Los conceptos de ritualidad, transposición de claves, claves, roles y el papel posibilitan describir lo planteado por Bourdieu con los conceptos de habitus y campo. Es justo este el punto de unión entre 
uno y otro autor, la materialización de la reforma (campo) y de la oralidad (habitus) presentados en la sala de audiencia de juicio oral (ritualidad). En palabras de Goffman la interacción o representación que se lleva a cabo en una sala de juicio oral depende del entorno de ese espacio: "como medios, herramientas para la personificación del individuo, que favorecen la representación dramatizada de la interacción social, esto incluye el mobiliario, el decorado, los equipos y otros elementos propios del trasfondo escénico, que proporcionan el escenario y utilería para el flujo de acción humana que se desarrolla ante, dentro o sobre él." (GOFFMAN. 2012, p. 36).

Goffman posibilitó que se observaran de manera analítica las audiencias y las prácticas adaptadas e interiorizadas de los operadores (claves y transposición de claves). En el lenguaje de Goffman los operadores son los actores de una representación de la vida social, en este caso en el campo jurídico. La representación, es siguiendo a Goffman, "una disposición de cosas que transforman a un individuo en un actor", en función de que "el individuo se presenta y presenta su actividad ante otros [...] como una forma guiada y controlada de la impresión que los otros se forman de él." (GOFFMAN, 2006, p.13-31).

Cada actor (operador jurídico), construye su papel, es decir la función o el rol del Juez, MP o ADP que se desenvuelven en los juicios orales. La interacción entre todos los involucrados solo es posible en el sistema acusatorio, es por ello que hacer un análisis donde se entrelazan los intereses, las prácticas antiguas, las nuevas habilidades o reconfiguración de habitus, la reconfiguración del escenario, es útil para observar si en realidad los operadores actúan apegados a lo que estipula la ley. 


\section{Conclusiones}

El cambio en el sistema de justicia en México generó que las instituciones que imparten justicia y los operadores perciban de manera distinta la justicia. Los gobiernos se esforzaron por establecer la confianza en las instituciones de procuración e impartición de justicia mediante una reforma estructural, con el objetivo de erradicar los vicios que los operadores tenían en el sistema inquisitivo.

La oralidad es el eje central del nuevo sistema, es la guía para la interacción de todos los involucrados en el proceso. Este eje trajo consigo que el proceso se vuelva para algunos jueces un proceso humanizado, es decir, la oralidad provoca que los operadores sean visibles. También se visibiliza a la víctima y al imputado, con el fin de lograr mayor equidad en el proceso. Con base en el respeto de los derechos humanos para las dos partes.

En las audiencias de juicio oral es donde se ponen en juego los capitales que los operadores adquirieron por medio de los cursos de capacitación y también se observan los capitales acumulados en sus trayectorias judiciales. Prácticas nuevas que se cruzan con prácticas antiguas. Es aquí donde se puede observar la importancia de la oralidad, pues ahora es necesario ratificar las pruebas frente al Juez, al ADP y al imputado. Todo el contenido de la carpeta de investigación tiene que presentarse de manera oral y física en la audiencia. Ahora el sistema se basa en la presunción de inocencia del imputado, y es el MP quien tiene que demostrar la culpabilidade del detenido. En el sistema se partía de la presunción de culpabilidad, es decir, el imputado era culpable y él tenía que demostrar lo contrario.

Es posible que la oralidad pueda transformar la justicia y sus prácticas. La certeza jurídica se modificó entre el nuevo y viejo sistema. Antes se basaba en lo escrito y el proceso era hermético, ahora el proceso es público y con la posibilidad de observar todas y cada 
una de las pruebas de manera oral, esto es lo que desde la percepción de los operadores es mejor en el sistema oral; es más transparente y menos susceptible a la corrupción.

Es claro que por el tiempo que duró el sistema inquisitivo a los operadores les cueste interiorizar las nuevas prácticas impuestas por la reforma, pero también se puede observar que están dispuestos al cambio.

Lo que quedaría por analizar es si es posible que el nuevo sistema mediante la oralidad pueda generar un sistema más garantista que se apegue al debido proceso y por ende al respeto de los derechos humanos tanto para la víctima como para el imputado, y si este es el camino indicado para que la justicia en México genere mayor confianza y transparencia en su proceso.

\section{REFERÊNCIAS}

ALVARADO MENDOZA, Arturo. El tamaño del infierno. Un estudio sobre la criminalidad en la Zona Metropolitana de la Ciudad de México. México: El Colegio de México, 2012.

BOURDIEU, P. El sentido práctico. Buenos Aires: Siglo XXI, 2007.

BOURDIEU, P.; WACQUANT, L. Una invitación a la sociología reflexiva. Buenos Aires: Siglo XXI, 2005.

GOFFMAN, Erving. Frame analysis: los marcos de la experiencia. Madrid: CIS/Siglo XXI, 2006.

GOFFMAN, Erving. La presentación de la persona en la vida cotidiana. Buenos Aires: Amorrortu, 2012.

MÉXICO. Congreso General. Código Nacional de Procedimientos Penales. Diario Oficial de La Federación, 5 febr. 2014. Disponible em: <http://dof.gob.mx/nota_ detalle.php?codigo=5334903\&fecha=05/03/2014>. Aceso em: 15 maio 2015.

MÉXICO. Plan Nacional de Desarrollo 2006-2012. 2012. Disponível em: <http://pnd. calderon.presidencia.gob.mx/index.php?page=procuracionjusticia $>$. Aceso em: 15 maio 2015. 
MÉXICO. Secretaría Técnica del Consejo de Coordinación para la Implementación del Sistema de justicia Penal. ¿Que es la SETEC? Disponible em: <http://www.setec. gob.mx/es/SETEC/SETEC_MASTER>. Aceso em: 15 maio 2015.

ZEPEDA LECUONA Guillermo. Crimen sin castigo. Procuración de justicia penal y ministerio público. México: Fondo de Cultura Económica/CIDAC, 2011. 